\title{
Emergence of a Novel Recombinant Norovirus GII.P16-GII.12 Strain Causing Gastroenteritis, Alberta, Canada
}

\author{
Kanti Pabbaraju, Anita A. Wong, \\ Graham A. Tipples, Xiaoli-Li Pang
}

We identified a novel recombinant GII.P16-GII.12 norovirus associated with epidemic and endemic gastroenteritis during March 1, 2018-February 12, 2019, in Alberta, Canada. GIl.12 viruses have not been detected in Alberta since 2000. Comparing the full genome of this strain to previously published sequences revealed this virus to be a novel recombinant strain.

$\mathrm{N}$ orovirus is the leading cause of epidemic and endemic acute gastroenteritis (AGE) worldwide. Norovirus can evade the host immune response by accumulating mutations that have a biological advantage by antigenic drift (1). In addition, recombination at the junction of open reading frame (ORF) 1 and 2 can result in the circulation of a novel strain.

In the past 2 decades, emerging genetic clusters of norovirus GII.4 were associated with epidemics in Alberta, Canada. Multiple GII.4 viruses were associated with epidemics during the 2000s. Since 2010, a single variant, GII.4 Sydney, has been the predominant virus (2-5). A recombinant GII.P16-GII.4 Sydney strain emerged in July 2015 and caused $72 \%$ of the outbreaks in the winter of $2017-18$ (2). A small wave of activity with the Kawasaki GII.17 and GII.P16-GII.2 strains was seen during 2016-2017, but they did not predominate.

The Alberta Molecular Surveillance Program includes genotyping of 1 norovirus-positive stool sample from each outbreak for the early detection of novel strains. During March 1, 2018-February 12, 2019, we report the detection of a novel recombinant GII.P16-GII.12 that was identified in May 2018 and caused AGE outbreaks and sporadic cases in children $<6$ years of age.

\section{The Study}

Public health officials and the Public Health Laboratory (ProvLab) in the province of Alberta use established

Author affiliations: Public Health Laboratory (ProvLab), Alberta Public Laboratories, Edmonton, Alberta, Canada (K. Pabbaraju,

A.A. Wong, G.A. Tipples, X.-L. Pang); University of Alberta,

Edmonton, Alberta, Canada (G.A. Tipples, X.-L. Pang)

DOI: https://doi.org/10.3201/eid2508.190059 protocols to investigate all suspected AGE outbreaks. Stool samples are tested for norovirus genogroups I and II using a real-time reverse transcription PCR (4). An outbreak includes $\geq 2$ epidemiologically linked AGE cases with $\geq 1$ norovirus-positive sample. ProvLab genotyped 1 norovirus-positive sample from each outbreak and those from children with sporadic AGE by using the dual polymerasecapsid genotyping protocol (6). In our study, 72/108 (67\%) AGE outbreaks had test results positive for norovirus, of which $5(7 \%)$ were identified as norovirus GI, $66(92 \%)$ as GII, and $1(1 \%)$ as GI and GII (Table 1). During the same period, 94/755 (12\%) AGE cases in children had test results positive for norovirus, 6 as GI and 88 as GII.

We genotyped 64/72 (89\%) outbreaks and 74/94 (79\%) sporadic cases and detected diverse genotypes and different monthly trends (Table 2; Figure 1). GII.P16-GII.4 Sydney 2012 was the predominant strain followed by GII.P16-GII.12 in both outbreaks and sporadic AGE, but distribution of other genotypes was different, most notably GII.P16-GII.2 and GII.P12-GII.3 strains were detected in the sporadic cases but not in outbreaks (Table 2).

The GII.P16-GII.2 strain emerged in 2016 and caused a large norovirus epidemic in Asia $(7,8)$, followed by a small wave in Alberta in winter 2016-17 (2), but this strain was not detected in outbreaks during our study. Of note, the emerging GII.P16-GII.12 strain became the second most predominant strain with increasing cases, especially in sporadic AGE, after November 2018 (Figure 1, panel B). This strain caused $80 \%(8 / 10)$ of outbreaks in long-term care facilities and 2 in hospital acute-care units. Of the 17 sporadic cases associated with this strain, we retrieved patient information for 14 cases; 12 were hospitalized, 1 was seen in the emergency department, and 1 was an outpatient. The number of hospitalized patients probably indicates more severe disease associated with this strain. However, because the number of cases was small, further study and caution in interpretation of disease severity is warranted.

We sequenced the near-complete genome of the novel GII.P16-GII.12 strain from 2 different outbreaks by Sanger sequencing using primers designed in-house (available upon request). We performed contig assembly with Seqscape v2.7 (Advanced Biosystems, https://seqscape. software.informer.com/2.7) and sequence alignments with 
Novel Recombinant Norovirus GII.P16-GII.12, Canada

Table 1. Investigation of outbreaks of acute gastroenteritis, March 1, 2018-February 12, 2019, Alberta, Canada

\begin{tabular}{lccccc}
\hline Month & Outbreaks & $\begin{array}{c}\text { Norovirus-positive, } \\
\text { no. (\%) }\end{array}$ & GI & GII & GI and GII \\
\hline Mar & 16 & $11(69)$ & 1 & 10 & 0 \\
Apr & 9 & $8(89)$ & 1 & 7 & 0 \\
May & 4 & $2(50)$ & 1 & 1 & 0 \\
Jun & 2 & $0(0)$ & 0 & 0 & 0 \\
Jul & 3 & $1(33)$ & 0 & 1 & 0 \\
Aug & 3 & $1(33)$ & 0 & 0 & 1 \\
Sep & 3 & $1(33)$ & 0 & 1 & 0 \\
Oct & 4 & $3(75)$ & 0 & 3 & 0 \\
Nov & 12 & $8(67)$ & 0 & 8 & 0 \\
Dec & 26 & $17(65)$ & 0 & 17 & 0 \\
Jan & 21 & $15(71)$ & 2 & 13 & 0 \\
Feb & 5 & $5(100)$ & 0 & 5 & 0 \\
\hline Total & 108 & $72(67)$ & 5 & 66 & 1 \\
\hline
\end{tabular}

ClustalW (http://www.clustal.org/clustal2). For phylogenetic analysis, we inferred evolutionary history by using the neighbor-joining method. We computed evolutionary distance by using the maximum composite likelihood model for nucleotide sequences and the Poisson model for amino acid sequences. We performed a bootstrap test by using 1,000 replicates in MEGA6 (http://www.megasoftware.net) (9). We obtained 7,406 bp and submitted the sequences to GenBank (accession nos. MK355712-3).

We compared the ORF1 sequence to the RIVM (http:// www.rivm.nl/mpf/norovirus/typingtool) and National Center for Biotechnology Information (http://www.ncbi.nlm. nih.gov) databases. The closest sequence match was from GII.P16-GII.4 Sydney 2012 (accession no. LC175468), the current predominant genotype worldwide $(6,10)$. At the nucleotide level, ORF1 of MK355712 shared $96.92 \%$ identity with the Sydney 2012 strain and MK355713 shared $96.98 \%$ identity. At the amino acid level, ORF1 of MK355712 shared $98.56 \%$ identity and MK355713 shared $98.65 \%$ identity with the Sydney 2012 strain. We constructed a phylogenetic tree comparing the near full-length ORF1 of the strains we report with the GII.P16 sequence associated with the viral protein (VP) 1 capsid region from different genotypes (Figure 2). The ORF1 clustered with the ORF1 from contemporary strains including GII.4
Table 2. Genotyping results for outbreak and sporadic cases of acute gastroenteritis, March 1, 2018-February 12, 2019, Alberta, Canada

\begin{tabular}{|c|c|c|c|}
\hline Characteristics & Outbreak & Sporadic & Total \\
\hline No. cases & 72 & 94 & 166 \\
\hline No. (\%) genotyped & $64(89)$ & $74(79)$ & $138(83)$ \\
\hline \multicolumn{4}{|l|}{ GI genotypes, no. cases } \\
\hline GI.P2-GI.2 & 0 & 1 & 1 \\
\hline GI.P3-GI.3 & 3 & 2 & 5 \\
\hline GI.Pb-GI.6 & 1 & 0 & 1 \\
\hline GI.P6-GI.6* & 1 & 0 & 1 \\
\hline GI.P7-GI.7 & 0 & 1 & 1 \\
\hline GI.PUnknown-GI.7 & 1 & 0 & 1 \\
\hline \multicolumn{4}{|l|}{ Gll genotypes, no. cases } \\
\hline GII.P4-GII.4 & 1 & 1 & 2 \\
\hline GII.P7-GII.6 & 1 & 0 & 1 \\
\hline GII.P7-GII.14* & 1 & 0 & 1 \\
\hline GII.P12-GI.3 & 0 & 4 & 4 \\
\hline GII.P16-GII.2 & 0 & 2 & 2 \\
\hline GII.P16-GII.4 & 46 & 44 & 90 \\
\hline GII.16-GII.12 & 10 & 17 & 27 \\
\hline GII.P-unknown_GII.12 & 0 & 2 & 2 \\
\hline
\end{tabular}

Sydney, GII.2, and the recently described GII.3 and GII.1 sequences (accession nos. KY887597 and MG572182), but our sequences lie on an independent branch.

GII.P16 sequences from this study clustered with GII.P16 sequences that form a previously reported distinct monophyletic clade with a common ancestor from 2013 (10) but lie on an independent branch (data not shown). Others have described the GII.P16 sequences belonging to this clade as containing nonsynonymous substitutions in ORF1 along the branch leading to the common ancestor of the GII.P16-GII.4 Sydney 2012/GII.3 clade, several of which occur close to positions known to affect polymerase function and transmission (10). We saw amino acid changes in our sequences that were also noted in the GII.Pe and GII.P16 polymerase of GII.4 Sydney viruses, including D173E, G163A, L337M, and S502N, but we did not see the K1646R unique to GII.P16 associated with GII.4 Sydney. We noted some of the point mutations in our sequences that have been described on the polymerase surface of novel GII.P16 strains, including
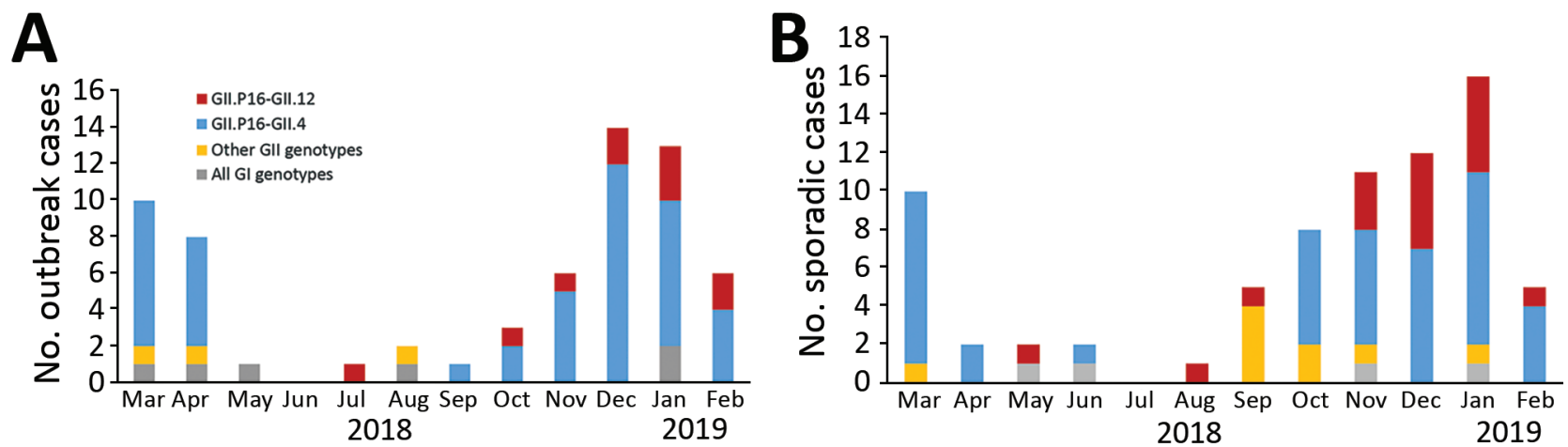

Figure 1. Monthly trends of norovirus genotypes for outbreak (A) and sporadic (B) cases of acute gastroenteritis in Alberta, Canada, during March 2018-February 2019. Genotypes included under other are listed in Table 2. 


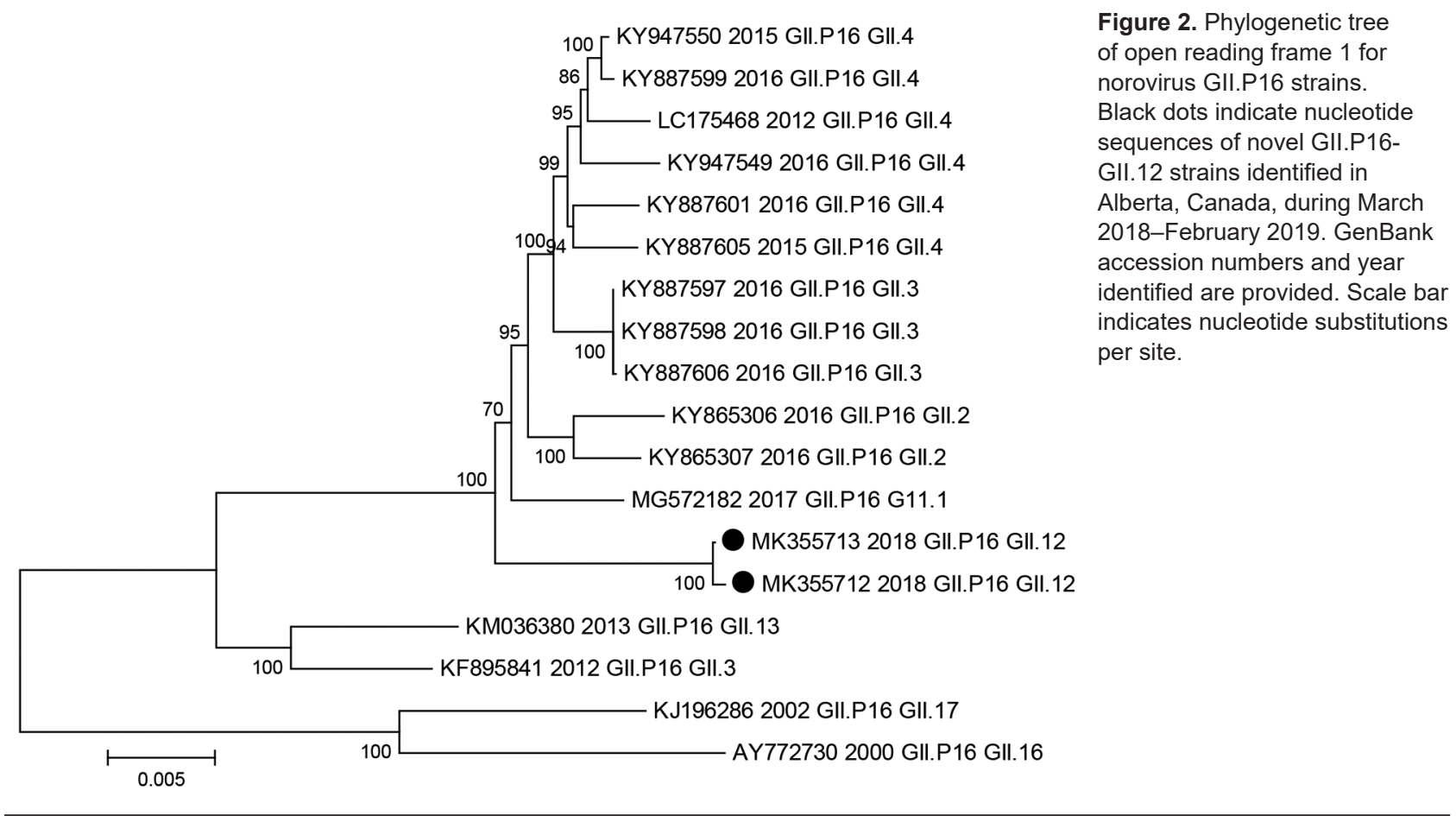

Figure 2. Phylogenetic tree open reading frame 1 for accession numbers and year identified are provided. Scale bar ndicates nucleotide substitutions per site.
K357Q, T360A, and V332I $(6,7,10,11)$, which caused reemergence of a new cluster. Because we did not see all the known mutations, effects of these amino acid changes will require further investigation.

We compared ORF2 and ORF3 to the GenBank database using BLAST (https://blast.ncbi.nlm.nih.gov/Blast. cgi) and found the closest sequence matches are contemporary strains described in Taiwan (12), Australia (13), and the United States $(14,15)$. Identity with these sequences were $93 \%-94 \%$ at the nucleotide level and $98 \%-99 \%$ at the amino acid level in ORF2 and $84 \%-85 \%$ at the nucleotide level and $86 \%-87 \%$ at the amino acid level in ORF3. Identity with the prototype Wortley strain (accession no. AJ277618) was lower. Our sequences demonstrated amino acid changes reported in the contemporary GII.12 strains, including A22V, I47V, and S465T, but we did not see the N392S described in all sequences of the GII.12 strain cluster in our sequence (15). The P2 region of VP1 has the antigenic and histoblood group antigen attachment sites, and 3 unique amino acid substitutions, including N350D, A352T, and $\mathrm{I} 361 \mathrm{~V}$ were seen in this region. Studies to explore whether these mutations play a role in binding profiles and immunity will help us understand if this virus is under selective pressure, which can give rise to novel variants.

\section{Conclusions}

We describe a novel recombinant GII.P16-GII.12 norovirus strain identified in Alberta, Canada. Although the GII.P16GII.4 strain is still predominant, this novel strain seems to be playing a role in both outbreaks and sporadic cases in young children. Continued surveillance and prompt genotyping are critical to monitor the emergence and prevalence of novel norovirus strains.

\section{Acknowledgments}

We thank Chen Liu for managing the samples for this study. We also thank the staff of the Public Health Laboratory (ProvLab), especially the virology and molecular departments, for performing norovirus testing; the collaborative teams participating in outbreak investigations in Alberta, Northwest Territories, Yukon, and Nunavut; and the members of the ProvLab Outbreak Investigation Committee.

This work was supported by the Public Health Laboratory (ProvLab), Alberta, Canada, and Alberta Health, Canada.

\section{About the Author}

Dr. Pabbaraju is a laboratory scientist at the Public Health Laboratory (ProvLab), Alberta, Canada. Her research focuses on the development of diagnostic tests for viral and bacterial pathogens and studies on the epidemiology of viruses.

\section{References}

1. Siebenga JJ, Vennema H, Renckens B, de Bruin E, van der Veer B, Siezen RJ, et al. Epochal evolution of GGII.4 norovirus capsid proteins from 1995 to 2006. J Virol. 2007;81:9932-41. http://dx.doi.org/10.1128/JVI.00674-07

2. Hasing ME, Lee BE, Qiu Y, Xia M, Pabbaraju K, Wong A, et al. Changes in norovirus genotype diversity in gastroenteritis outbreaks in Alberta, Canada: 2012-2018. BMC Infect Dis. 2019;19:177. http://dx.doi.org/10.1186/s12879-019-3792-y 
3. Hasing ME, Lee BE, Preiksaitis JK, Tellier R, Honish L, Senthilselvan A, et al. Emergence of a new norovirus GII.4 variant and changes in the historical biennial pattern of norovirus outbreak activity in Alberta, Canada, from 2008 to 2013. J Clin Microbiol. 2013;51:2204-11. http://dx.doi.org/10.1128/ JCM.00663-13

4. Pang XL, Preiksaitis JK, Wong S, Li V, Lee BE. Influence of novel norovirus GII.4 variants on gastroenteritis outbreak dynamics in Alberta and the Northern Territories, Canada between 2000 and 2008. PLoS One. 2010;5:e11599. http://dx.doi.org/10.1371/journal.pone.0011599

5. Siebenga JJ, Vennema H, Zheng DP, Vinjé J, Lee BE, Pang XL, et al. Norovirus illness is a global problem: emergence and spread of norovirus GII.4 variants, 2001-2007. J Infect Dis. 2009;200:802-12. http://dx.doi.org/10.1086/605127

6. Cannon JL, Barclay L, Collins NR, Wikswo ME, Castro CJ, Magaña LC, et al. Genetic and Epidemiologic Trends of Norovirus Outbreaks in the United States from 2013 to 2016 Demonstrated Emergence of Novel GII.4 Recombinant Viruses. J Clin Microbiol. 2017;55:2208-21. http://dx.doi.org/10.1128/ JCM.00455-17

7. Ao Y, Wang J, Ling H, He Y, Dong X, Wang X, et al. Norovirus GII.P16/GII.2-associated gastroenteritis, China, 2016. Emerg Infect Dis. 2017;23:1172-5. http://dx.doi.org/10.3201/ eid2307.170034

8. Liu LT, Kuo TY, Wu CY, Liao WT, Hall AJ, Wu FT. Recombinant GII.P16-GII.2 norovirus, Taiwan, 2016. Emerg Infect Dis. 2017;23:1180-3. http://dx.doi.org/10.3201/ eid2307.170212

9. Tamura K, Stecher G, Peterson D, Filipski A, Kumar S. MEGA6: Molecular Evolutionary Genetics Analysis version 6.0. Mol Biol Evol. 2013;30:2725-9. http://dx.doi.org/10.1093/molbev/ mst197

10. Ruis C, Roy S, Brown JR, Allen DJ, Goldstein RA, Breuer J. The emerging GII.P16-GII.4 Sydney 2012 norovirus lineage is circulating worldwide, arose by late-2014 and contains polymerase changes that may increase virus transmission. PLoS One. 2017;12:e0179572. http://dx.doi.org/10.1371/ journal.pone.0179572

11. Tohma K, Lepore CJ, Ford-Siltz LA, Parra GI. Phylogenetic analyses suggest that factors other than the capsid protein play a role in the epidemic potential of GII.2 norovirus. mSphere. 2017;2:e00187-17. PubMed http://dx.doi.org/10.1128/ mSphereDirect.00187-17

12. Tsai CN, Lin CY, Lin CW, Shih KC, Chiu CH, Chen SY. Clinical relevance and genotypes of circulating noroviruses in northern Taiwan, 2006-2011. J Med Virol. 2014;86:335-46. http://dx.doi.org/10.1002/jmv.23728

13. Eden JS, Bull RA, Tu E, McIver CJ, Lyon MJ, Marshall JA, et al. Norovirus GII.4 variant $2006 \mathrm{~b}$ caused epidemics of acute gastroenteritis in Australia during 2007 and 2008. J Clin Virol. 2010;49:265-71. http://dx.doi.org/10.1016/j.jcv.2010.09.001

14. Takanashi S, Wang Q, Chen N, Shen Q, Jung K, Zhang Z, et al. Characterization of emerging GII.g/GII.12 noroviruses from a gastroenteritis outbreak in the United States in 2010. J Clin Microbiol. 2011;49:3234-44. http://dx.doi.org/10.1128/ JCM.00305-11

15. Vega E, Vinjé J. Novel GII.12 norovirus strain, United States, 2009-2010. Emerg Infect Dis. 2011;17:1516-8. http://dx.doi.org/ $10.3201 /$ eid 1708.110025

Address for correspondence: Xiao-Li Pang, Public Health Laboratory (ProvLab), Alberta Public Laboratories, University of Alberta Hospital, WMC 2B4.58, 8440-112 Street, Edmonton, AB T6G 2J2, Canada; email: xiao-li.pang@albertapubliclabs.ca

\section{EID Podcast: Developing Biological Reference Materials to Prepare for Epidemics}

Having standard biological reference materials, such as antigens and antibodies, is crucial for developing comparable research across international institutions. However, the process of developing a standard can be long and difficult.

In this EID podcast from February 2019, Dr. Tommy Rampling, a clinician and academic fellow at the Hospital for Tropical Diseases and University College in London, explains the intricacies behind the development and distribution of biological reference materials.

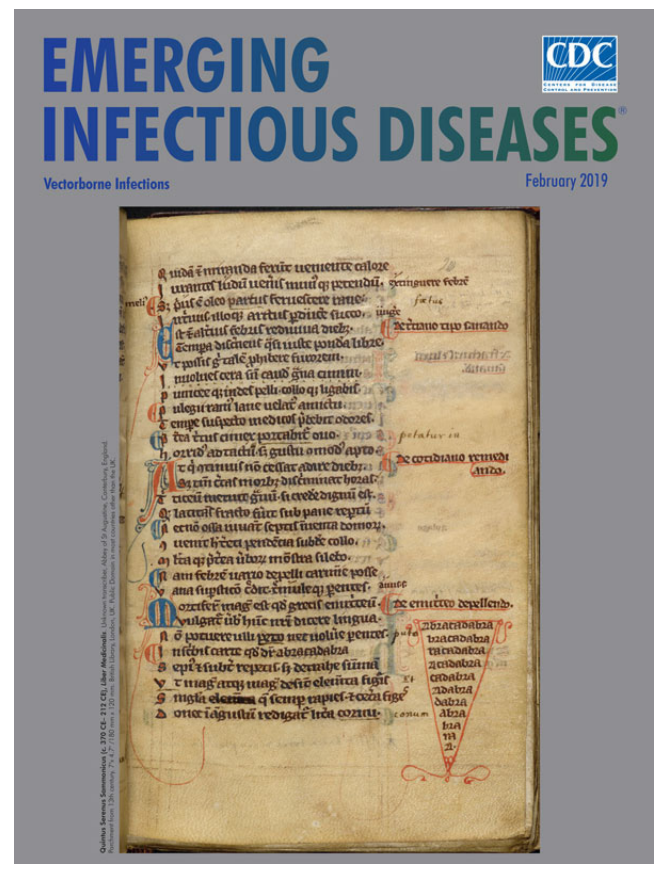

\section{Visit our website to listen: https://go.usa.gov/xy8bg}

\section{EMERGING INFECTIOUS DISEASES}

